\title{
Impact of flux jumps in future colliders
}

\author{
J. Coello de Portugal@, R. Tomás, L. Fiscarelli®, D. Gamba®, and M. Martino \\ CERN, Geneva 1211, Switzerland
}

(Received 23 October 2019; published 2 January 2020)

\begin{abstract}
Type-II superconductors, like the $\mathrm{Nb}_{3} \mathrm{Sn}$ used in the new HL-LHC quadrupole triplet and $11 \mathrm{~T}$ dipoles or FCC dipoles, are known to show an unstable behavior during magnetic-field ramps. This paper presents an analysis of the possible effects on the beam dynamics of this behavior of the magnets, with a focus on emittance blow-up.
\end{abstract}

DOI: 10.1103/PhysRevAccelBeams.23.011001

\section{INTRODUCTION}

The challenge of reducing the beam size at the interaction points has a very strong impact on the aperture requirements of the final focus system of colliders. For HLLHC, it is expected to have a $\beta^{*}$ of down to $7.5 \mathrm{~cm}$ in certain configurations $[1,2]$. To achieve this, the $\beta$-function across the triplet region rises above $40 \mathrm{~km}$.

To accommodate such beam sizes, the current LHC triplet quadrupoles with $70 \mathrm{~mm}$ aperture will be replaced with a new triplet with $150 \mathrm{~mm}$ aperture [3]. To keep the required magnetic gradient seen by the beam, this new quadrupole package is longer than the one in the LHC and uses $\mathrm{Nb}_{3} \mathrm{Sn}$ superconductor technology, which allows to increase the magnetic gradient by about 50\% [4]. This technology is also going to be used in operation from LHC Run 3 by replacing 4 dispersion suppressor dipoles with shorter ones that use the $\mathrm{Nb}_{3} \mathrm{Sn}$ technology, to allow for the installation of additional collimators. The new technology is also expected to be used in all main dipoles and quadrupoles of the Future hadron-hadron Circular Collider (FCC-hh) [5].

Type I superconductors present superconductivity until they are subject to a magnetic field limit $H_{c}$, a point where the superconductivity is drastically destroyed. $\mathrm{Nb}_{3} \mathrm{Sn}$ is a type II superconductor, and the superconductivity is not lost at a single critical magnetic field $H_{c 1}$, but the material goes through a mixed state where the superconductivity is maintained until the magnetic field reaches a second critical magnetic field $H_{c 2}$ allowing for a significantly higher peak magnetic field. In this mixed state, the material does not show a complete Meissner effect [6] and the magnetic field

\footnotetext{
*Also at Universitat Politècnica de Catalunya.

Published by the American Physical Society under the terms of the Creative Commons Attribution 4.0 International license. Further distribution of this work must maintain attribution to the author(s) and the published article's title, journal citation, and DOI.
}

penetrates the bulk of the material. In this region, small spots of normal-conducting material start to form and the supercurrents circle around them forming vortexes that induce a quantized unit of magnetic flux. These local vortexes are called fluxoids. The dynamics of fluxoids in the material can cause local heating, starting a chain reaction called flux jump and either the material is capable of dissipating the heat as it spreads or the superconductivity is broken producing a quench of the magnet. During the flux jump, the magnetic flux through the magnet is disturbed, affecting the magnetic field seen by the beam.

In this paper the potential effect of these flux jumps on emittance (and orbit) will be studied based on magnetic measurement values. The results presented here ignore the effect of the presence of a transverse damper [7].

In Sec. II, recent measurements of the flux jumps on the magnetic field of short prototypes of the $11 \mathrm{~T}$ dipole and of the HL-LHC triplets are presented with focus on flux jump duration and magnitude. In Sec. III the mathematical approach used in this study to perform simulations of the effect of the flux jumps is presented. In Sec. IV, these simulations are discussed for the HL-LHC triplet with its possible impact on the luminosity of the machine. Section V shows that the flux jumps of the $11 \mathrm{~T}$ dipoles will have a negligible impact on performance. In Sec. VI a similar analysis is performed for the FCC-hh main dipoles.

\section{FLUX JUMP MEASUREMENTS}

Flux jumps measurements have been performed at CERN on prototypes of the 11 Tesla dipoles and of the MQX quadrupoles [8,9].

The effect of the flux jumps on the magnetic field as seen by the beam can be separated in two parts: the flux jump causing a change of inductance of the magnet [9] and thus directly the magnetic field, and the reaction of the feedback current-regulation loop of the power supply to the change of inductance, which can also be seen as a change of voltage across the magnet. 


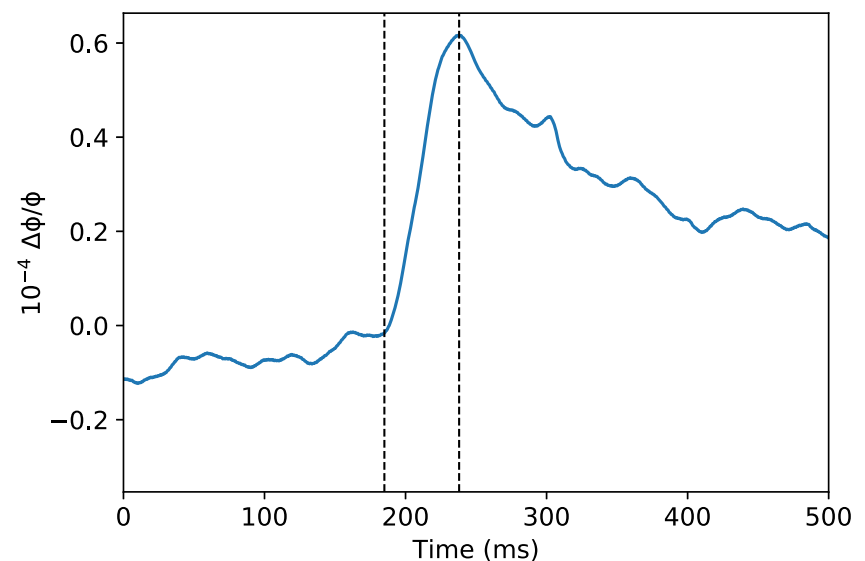

FIG. 1. Strongest flux jump observed during the measurement of relative magnetic flux error in an $11 \mathrm{~T}$ dipole model. The dashed lines limit the fast rise up time of the flux jump.

\section{A. Error in the magnetic field}

The effect of the flux jumps on the field itself has been measured on the MBHSP109 prototype of the $11 \mathrm{~T}$ dipole [8]. These measurements were performed by ramping the magnet at the nominal ramp rate $(10 \mathrm{~A} / \mathrm{s})$ and by measuring both the current error $(\Delta \mathrm{I})$ and the magnetic flux error inside the magnet $(\Delta \phi / \phi)$, both relative to their nominal values along the current ramp. The difference between these two values $(\Delta \phi / \phi-\Delta \mathrm{I} / \mathrm{I})$ measures the error in magnetic flux without the component coming from the error on the current. This measurement showed that the flux jumps happen mainly during the first half of the energy ramp of the magnet, between 2 and $3 \mathrm{kA}$ (corresponding to about 1.2 to $1.8 \mathrm{TeV}$ of beam energy) at a rate of about 4.4 flux jumps per second (at the nominal ramp rate). In Fig. 1, the relative magnetic flux error is shown for the strongest flux jump observed during the measurement. This displays the typical signature of a flux jump, consisting on a fast rise of the magnetic flux with a slower recovery toward the nominal value.

A statistical analysis is performed for the 145 flux jumps that are clearly distinguishable from the noise, Fig. 2. For each flux jump the rise time and the change of magnetic flux are recorded. The average rise time of the flux jumps is $46 \pm 15 \mathrm{~ms}$, or $506 \pm 165$ turns at the LHC revolution frequency and the average relative error in flux is $(0.2 \pm 0.1) \times 10^{-4}$. There is an approximately linear relation between the length of the flux jumps and the relative flux error it produces, suggesting that the slope of the flux variation is more or less constant.

\section{B. Reaction of the feedback regulation loop}

Power converters supply particle accelerator magnets with very high-precision current thanks to a dedicated feedback regulation loop. Such feedback loop is often composed of nested ones: the external one regulates the
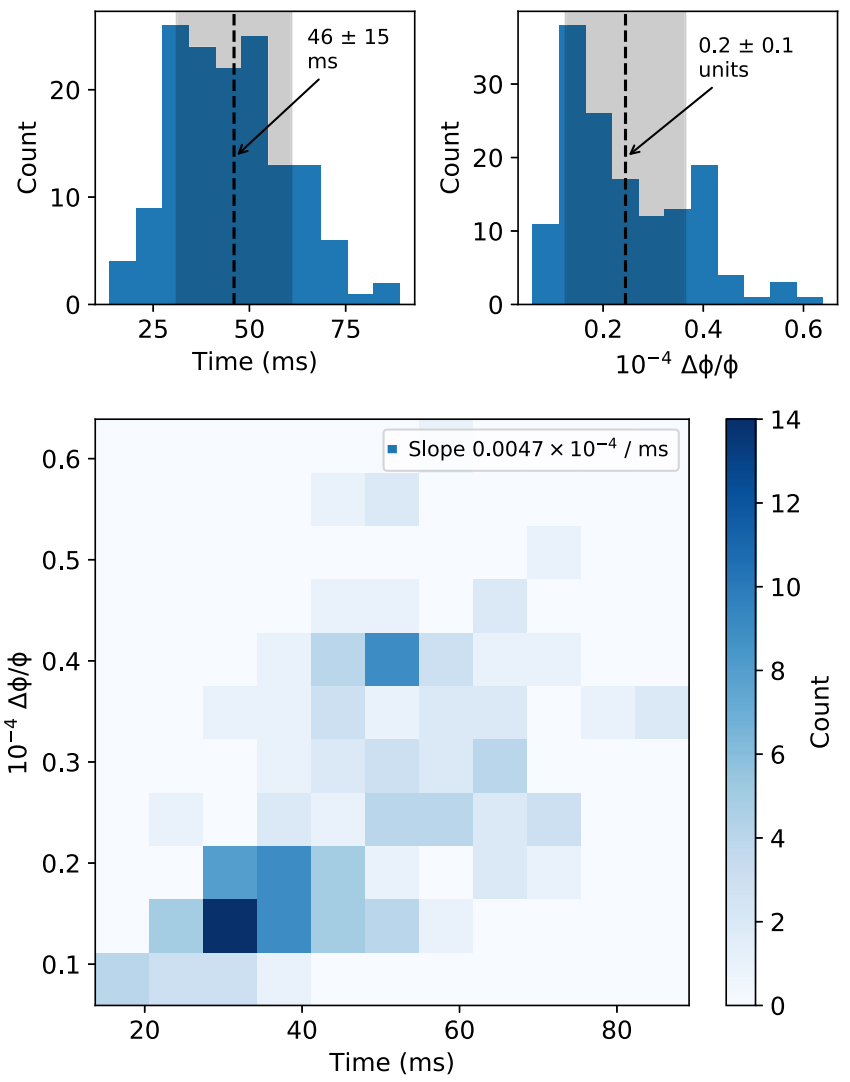

FIG. 2. Statistics of 145 manually selected flux jumps during the measurement of the magnetic flux error in the MBHSP109 model of the $11 \mathrm{~T}$ dipole. The top left plot shows the distribution of the rise times of the selected flux jumps. The top right plot shows the distribution of the magnetic flux change that the flux jumps produce. In the bottom plot the distribution of magnetic flux error versus rise time is shown.

current and the internal one controls the voltage. Both loops react to flux jumps hence introducing an additional error in the current and therefore in the magnetic field.

The internal regulation loop measures the voltage across a set of magnets and reacts to the voltage changes produced by the flux jumps, introducing an additional error in the current and therefore in the magnetic field. Measurements of the voltage across the MQXFS4b model of the HL-LHC triplet quadrupoles have been performed and a simplified model of the effect able to match this measurement (by matching simulated and measured voltage spectra) has been developed. Such a model allows to predict the reaction of a simulated HL-LHC triplet power converter, ignoring the effect of the trim circuits. A realistic configuration of the feedback regulation loop, whose bandwidth is limited to a few hertz, has been assumed for this study. Further details of these measurements and simulations are given in $[9,10]$. These measurements, and therefore the simulation results, should be considered pessimistic as they were performed at a ramp rate of $51 \mathrm{~A} / \mathrm{s}$, while the nominal ramp rate for HLLHC triplet quadrupole magnets is $14.6 \mathrm{~A} / \mathrm{s}$ and the flux 

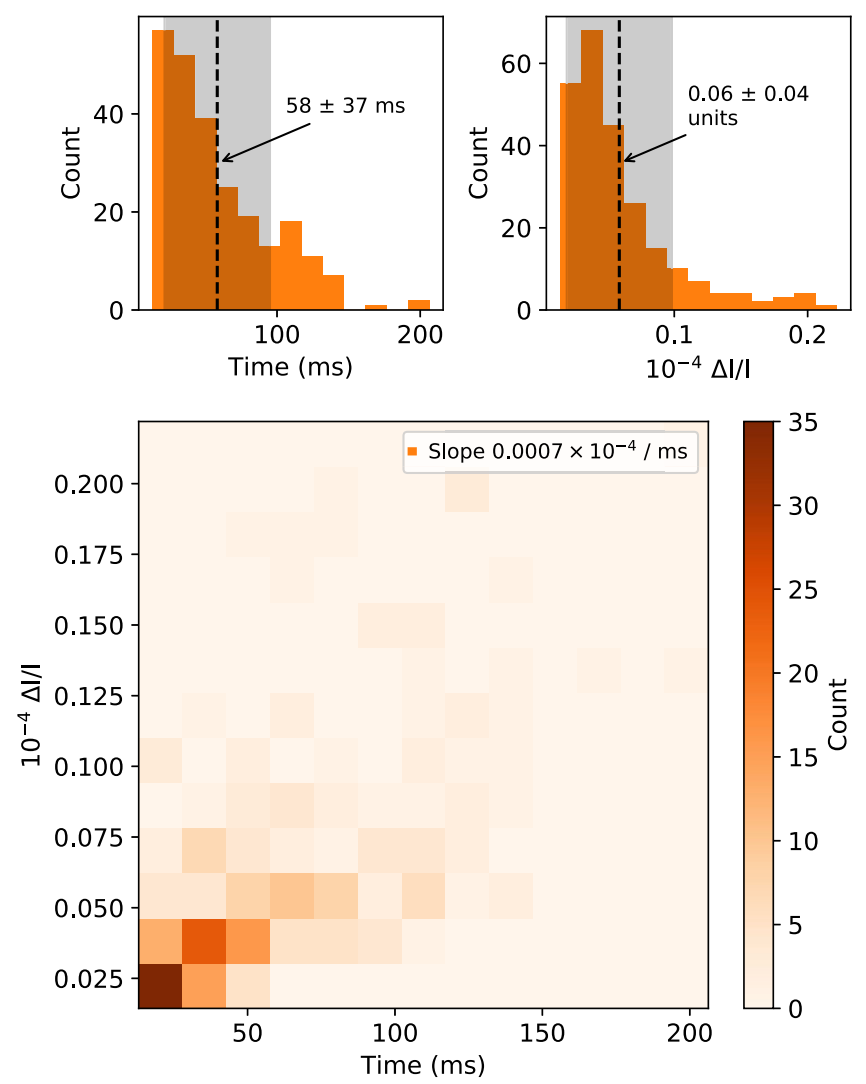

FIG. 3. Statistics of the simulation of the current error produced by the regulation loop. The top left plot shows the distribution of the rise time of the current error. The top right plot shows the distribution of the total magnitude of the current error. In the bottom plot, the distribution of the length of the current errors versus the current error magnitude is shown.

jumps are likely to happen more often for faster current changes.

Figure 3 shows a manual selection of 244 flux jumps (again, those visible above the noise level) as produced by the inductance jump model presented in [9]. As before, the rise time of the error and the total error in current produced during the jump are considered. The figure demonstrates how this effect is in general slower than the magnetic field jump, at $58 \pm 37 \mathrm{~ms}$ in length or $638 \pm 407$ turns at the LHC revolution frequency. This effect is in general a factor 4 weaker than the direct effect of the magnetic flux, at $(0.06 \pm 0.04) \times 10^{-4}$. As the heat map also shows in this case, there is an approximately linear relation between the current error and the duration of the flux jump.

The pessimistic side of the error bars presented in this section (instead of the average value) will be used in subsequent simulations, for both the duration and the strength of the jumps. The reference values used for the flux jump effect on the field itself will therefore be $0.3 \times$ $10^{-4}$ of field error and $31 \mathrm{~ms}$ of rise time. For the reaction of the regulation loop, $0.1 \times 10^{-4}$ relative field error and $21 \mathrm{~ms}$ of rise time will be used.
Additionally, flux jumps size is assumed equal for dipole and quadrupole magnets for now. Future measurements on the actual magnets (instead of prototypes) will define the correct values.

\section{EMITTANCE GROWTH}

As the beam traverses the triplet quadrupoles off-center due to crossing angle and separation bumps, the beam perceives both a dipolar kick (via feed-down) and a quadrupolar disturbance, i.e., a quadrupolar deformation of the phase space.

\section{A. Dipolar effect}

The dipolar case can be evaluated by computing the single-particle emittance change across the quadrupoles producing flux jumps. If $\Delta y$ and $\Delta y^{\prime}$ are the offsets with respect to the closed orbit, the geometric emittance growth compared with the reference particle can be written as:

$$
\Delta \epsilon_{\mathrm{geom}}=\gamma(s) \Delta y^{2}+2 \alpha(s) \Delta y \Delta y^{\prime}+\beta(s) \Delta y^{\prime 2},
$$

where $\alpha, \beta$, and $\gamma$ are the Twiss parameters and $s$ the longitudinal position. MAD-X [11] can be used to compute the difference in orbit and divergence from the nominal model and thus derive the normalised one-turn rise emittance growth $\Delta \epsilon_{1} / \epsilon=\gamma_{\text {rel }} \Delta \epsilon_{\text {geom }} / \epsilon$, relative to $\epsilon$, the design normalised emittance.

The flux jump evolution with time, as can be seen in Fig. 1, resembles a linear ramp with a slower decay. A simplified model of the flux jumps used here is shown in Fig. 4.

Using such a model we follow the derivations in [12] to compute emittance growth. In [12] a modulation with tune $Q_{D}$ is assumed on top of the linear ramp of the kick. In our simpler case the modulation tune is zero, $Q_{D}=0$. The equivalent quantity of Eq. (4) in [12] for our case is

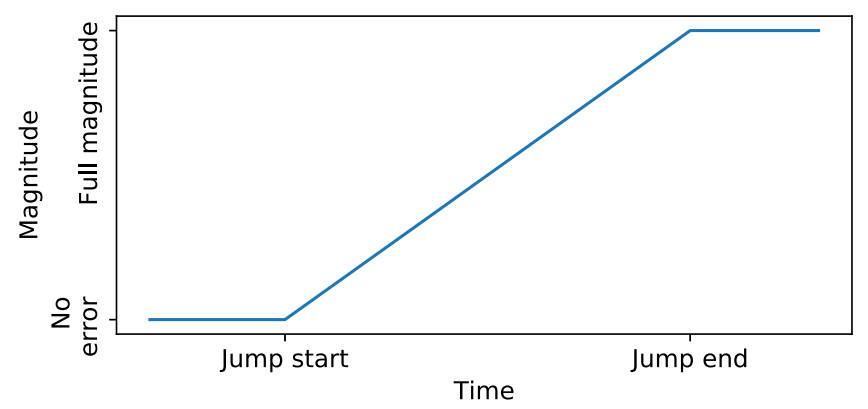

FIG. 4. Simplified flux jump event shape. It is modelled as a linear ramp toward the full amplitude of the jump. The duration of the ramp is on average $46 \mathrm{~ms}$ (506 turns in the LHC) for the flux jump on the magnetic field itself and $58 \mathrm{~ms}$ (638 turns in the LHC) for the reaction of the regulation loop. 


$$
\delta_{ \pm}=\frac{\sqrt{\Delta \epsilon_{1}}}{4 \sin (\pi Q)},
$$

with $Q$ the betatron tune. The oscillation amplitude in the normalized coordinate after a linear ramp of $N$ turns from Eq. (8) in [12] now becomes

$$
2 \delta_{ \pm} \frac{\sin (\pi Q N)}{N \sin (\pi Q)} .
$$

Therefore the total relative emittance change after a rise time of $N$ turns is given by

$$
\frac{\Delta \epsilon(N)}{\epsilon}=\frac{M \overline{\Delta \epsilon_{1} / \epsilon} \sin ^{2}(N \pi Q)}{4 N^{2} \sin ^{4}(\pi Q)},
$$

where $\overline{\Delta \epsilon_{1} / \epsilon}$ is the average relative one-turn emittance growth over $M$ magnets. For the sake of simplicity, all the quadrupoles of the triplet will be assumed equally likely to produce a flux jump. This formula oscillates with the number of turns and an envelope of the function can be used to stay in the worst-case scenario:

$$
\frac{\Delta \epsilon(N)}{\epsilon} \leq \frac{M \overline{\Delta \epsilon_{1} / \epsilon}}{4 N^{2} \sin ^{4}(\pi Q)}
$$

The model of the flux jumps used here (Fig. 4) neglects the effect of the slower recovery of the field which is significantly more adiabatic.

\section{B. Quadrupolar effect}

The fast current change in the quadrupoles also induces a quadrupolar deformation of the phase space that will also lead to emittance growth. This quadrupolar effect can be written as a mismatch of the $\alpha$-function after a quadrupolar kick. Defining

$$
p=\beta x^{\prime}+\alpha x,
$$

the quadrupolar kick in $p$ can be written as:

$$
\Delta p=\beta_{k} \Delta x^{\prime}=\beta_{k} \Delta k l x,
$$

with $\beta_{k}$ the $\beta$-function at the quadrupole and $\Delta k l$ the integrated quadrupolar error. This can then be written as a transformation in the $\alpha$-function:

$$
\begin{aligned}
& p \rightarrow p+\Delta p=\beta x^{\prime}+x\left(\alpha+\beta_{k} \Delta k l\right), \\
& \alpha \rightarrow \alpha+\beta_{k} \Delta k l .
\end{aligned}
$$

The emittance growth after an optics mismatch follows the equation [13]:

$$
1+\frac{\Delta \epsilon}{\epsilon}=\frac{1}{2}\left[\frac{\beta_{n}}{\beta_{k}}+\frac{\beta_{k}}{\beta_{n}}+\left(\frac{\alpha_{k}}{\beta_{k}}-\frac{\alpha_{n}}{\beta_{n}}\right)^{2} \beta_{n} \beta_{k}\right]
$$

with $\beta_{n}, \alpha_{n}$ the design $\beta$ and $\alpha$ functions and $\beta_{k}, \alpha_{k}$ the perturbed $\beta$ and $\alpha$ functions. In this case we only transform the $\alpha$-function so $\beta_{k}=\beta_{n}$ and $\alpha_{k}=\alpha_{n}+\beta_{k} \Delta k l$. Then, simplifying the equation and writing it in relative emittance growth terms:

$$
\frac{\Delta \epsilon}{\epsilon}=\frac{\left(\beta_{k} \Delta k l\right)^{2}}{2}
$$

This value is about $\frac{\Delta \epsilon}{\epsilon} \approx 7.5 \times 10^{-6}$ for the quadrupole with highest $\beta$-function (3324 m) at $\beta^{*}=1 \mathrm{~m}$, two orders of magnitude below the effect produced by the dipolar kick for the HL-LHC triplet and thus will be ignored. The $\beta_{k}^{2}$ term is likely to enhance the effect of the quadrupolar deformation of the beam shape in future machines, if the flux jumps are present in regimes where the $\beta$-function in the quadrupoles reaches some tens of kilometres.

\section{THE HL-LHC TRIPLET}

As the combined ramp and squeeze steps for HL-LHC are not yet defined in detail, in this study a baseline scenario of injection optics $\left(\beta^{*}=6 \mathrm{~m}\right)$ at $450 \mathrm{GeV}$ beam energy and a worst-case scenario of $\beta^{*}=1 \mathrm{~m}$ optics at $3200 \mathrm{GeV}$ are assumed. For reference, in Figs. 5 and 6 the $\beta$-functions and orbit across the triplet magnets of IR1 for the two considered scenarios are shown. The HL-LHC triplets in IR1 and IR5 are identical with the crossing angles in opposite planes. Here, a vertical crossing in IP1 is considered. Having horizontal crossing in IP1 and vertical in IP5 is under consideration for HL-LHC [14].

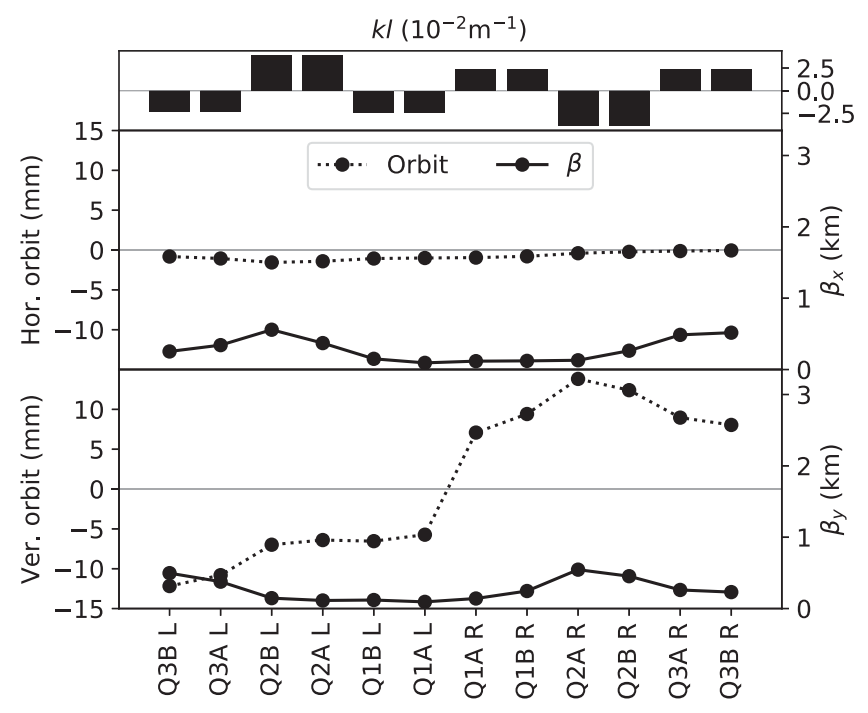

FIG. 5. Integrated quadrupolar strength $(k l)$, orbit and $\beta$ functions across the triplet of IR1 with injection optics. 


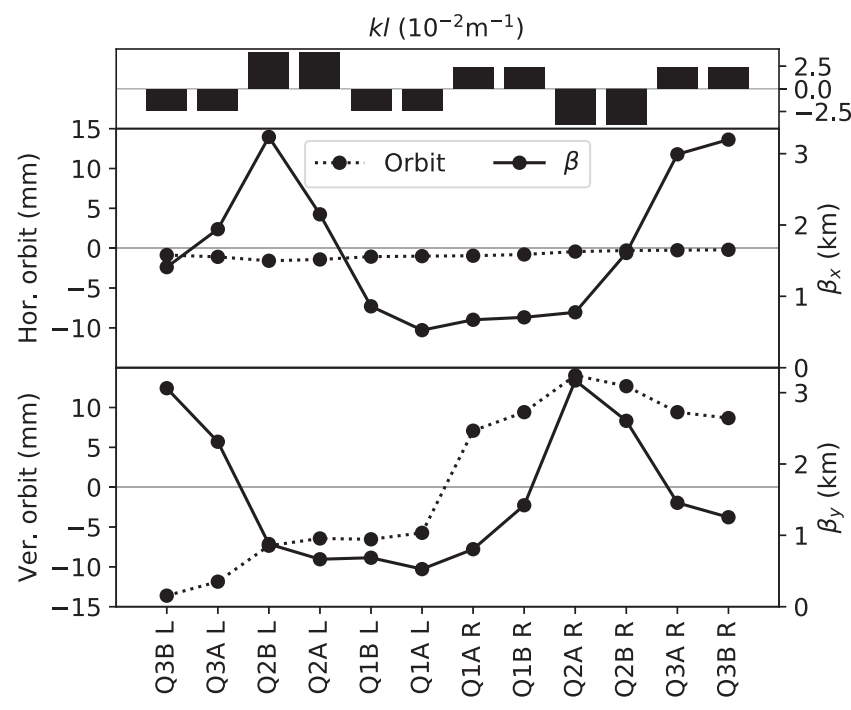

FIG. 6. Integrated quadrupolar strength $(k l)$, orbit and $\beta$ functions across the triplet of IR 1 with $\beta^{*}=1 \mathrm{~m}$ optics.

\section{A. Effect on the emittance}

For a one-turn flux jump, MAD-X simulations of the effect of a field disturbance of every magnet in the triplet is used to compute the beam position change and thus the normalized one-turn emittance growth $\Delta \epsilon_{1} / \epsilon$ relative to the normalized emittance of the HL-LHC at injection $\epsilon=\epsilon_{\mathrm{HL}-\mathrm{LHC}}=1.7 \mu \mathrm{m} \mathrm{[14].}$

The one-turn emittance growth produced by a flux jump of $0.3 \times 10^{-4}$ of the nominal field of each of the magnets of the triplet is shown in Fig. 7, with the expected dependency on the $\beta$-function and orbit in the quadrupoles.

Similarly, the impact of the reaction of the regulation loop on the emittance can be calculated. As already discussed, only the reaction of the main circuit of the triplet is considered here as it is unclear how the flux jumps will affect the different circuits of the triplet.

In Fig. 8 the relative emittance growth produced by a one-turn change of $0.1 \times 10^{-4}$ on the magnetic field of the triplets at the left and right of IP1 is shown. This effect is significantly smaller than that produced by the flux jump on the magnetic field of each individual quadrupole, as already shown in the measurements.

Figure 9 shows the results of Eqs. (4) and (5) for the emittance growth produced by the flux jumps in the magnetic flux itself for a range of rise times of $46 \pm$ $15 \mathrm{~ms}$ (341 to 670 turns) taken from the measurements shown in Fig. 2.

Same results for the reaction of the regulation loop are shown in Fig. 10 for a range of rise times $58 \pm 37 \mathrm{~ms}$ (231 to 1044 turns) as was presented in Fig. 3.

For the worst-case scenario, i.e., the shortest flux jumps using Eq. (5), an upper bound of the emittance blowup caused in a 20 minutes ramp can be given. Tables I and II show the number of flux jumps needed to produce a $1 \%$ emittance growth and the emittance growth that would be produced after a 20 minutes ramp at a flux jump rate of 4.4 events/s, for the flux jumps effect in the magnetic field itself and for the reaction of the regulation loop respectively. These results make the assumption that the flux
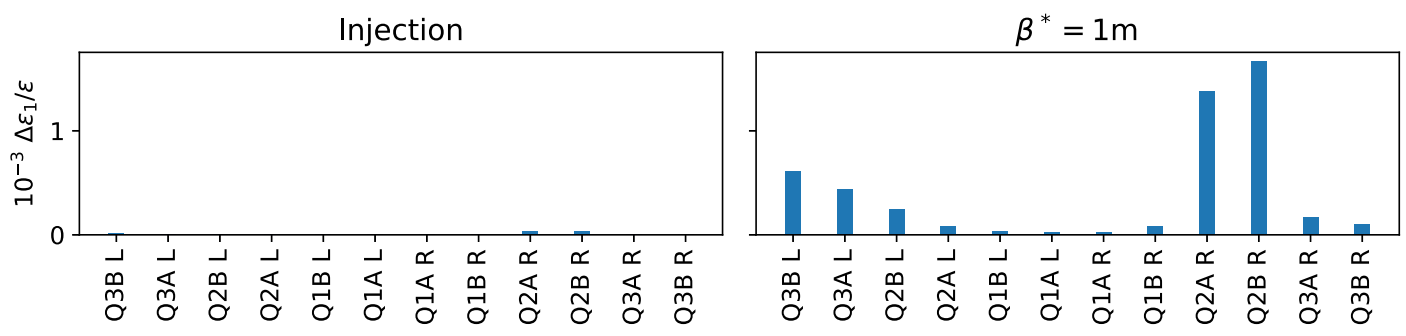

FIG. 7. Relative emittance growth produced by one-turn relative change of $0.3 \times 10^{-4}$ in the magnetic field of the quadrupoles of the HL-LHC triplet at injection (left) and $1 \mathrm{~m}$ optics (right) optics. The values follow the expected dependency on orbit and $\beta$-function.
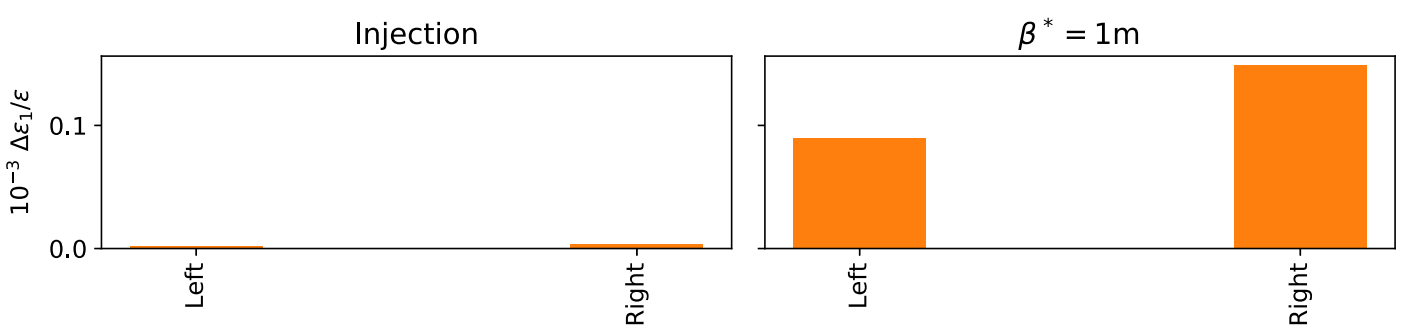

FIG. 8. Relative emittance growth produced by one-turn flux jumps causing a reaction of the regulation loop of the main circuit of each triplet at injection (left) and $1 \mathrm{~m}$ optics (right) optics, causing a relative strength change of $0.1 \times 10^{-4}$. It should be noted how this effect is significantly smaller than that produced by the flux jump in the field itself. 

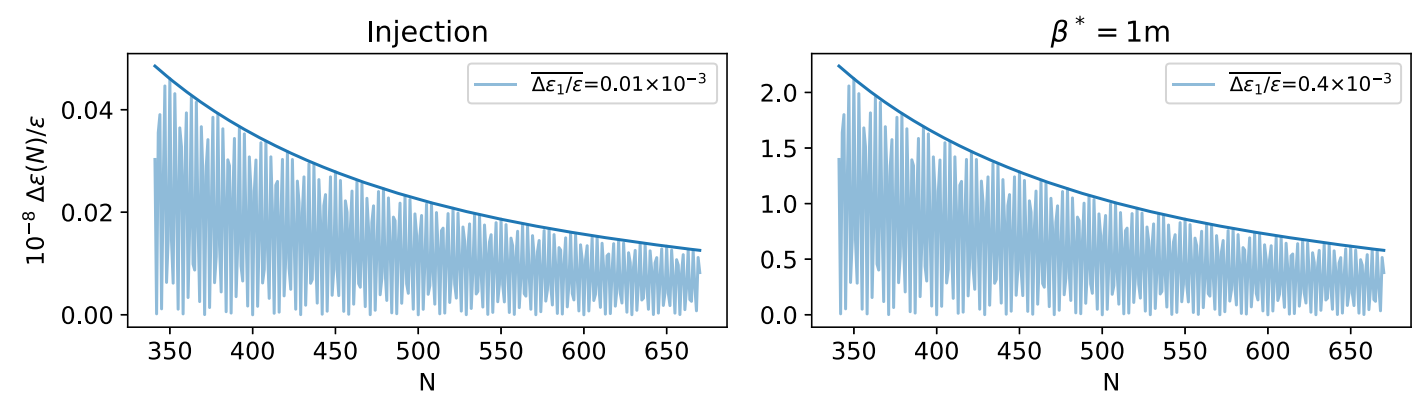

FIG. 9. Decay of the emittance growth with the length in turns (N) of the flux jumps effect on the magnetic flux itself, with an initial $\overline{\Delta \epsilon_{1} / \epsilon}$ of $8.8 \times 10^{-6}$ for injection and $4 \times 10^{-4}$ for $\beta^{*}=1 \mathrm{~m}$ (average of the values in Fig. 7). The clearer line shows the oscillating decay as shown on Eq. (4) and the darker line shows the pessimistic envelope described in Eq. (5).
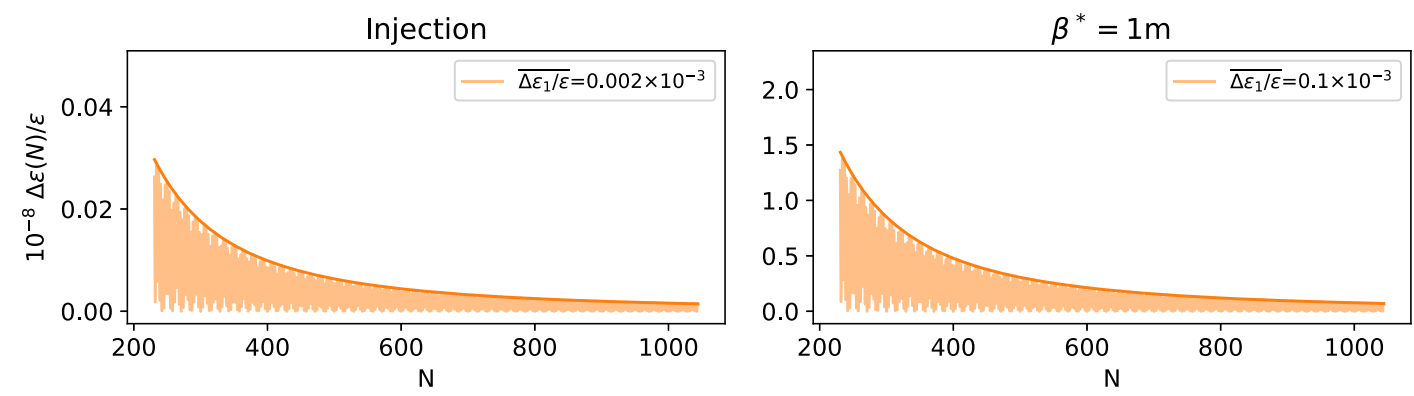

FIG. 10. Same results as in Fig. 9 but for the reaction of the regulation loop. In this case the initial $\overline{\Delta \epsilon_{1} / \epsilon}$ (average of the values in Fig. 8) is $2.5 \times 10^{-6}$ for injection and $1.2 \times 10^{-4}$ for $\beta^{*}=1 \mathrm{~m}$, factor 4 lower than for the previous effect. The larger spread of lengths causes the worst-case scenario of this effect to be about $60 \%$ of the direct effect on the magnetic flux.

jumps are equally likely and behave in the same way during the whole ramp, which is again pessimistic.

Even after these pessimistic assumptions, the worst-case emittance growth during the ramp is about a $0.1 \%$ o assuming that IR1 and IR5 are equivalent, producing emittance growths in the vertical and horizontal planes respectively.

In view of these results, it is safe to assume that the flux jumps will not have a harming effect on the emittance of HL-LHC.

\section{B. Effect on the orbit and tune noise}

Flux jumps in the HL-LHC triplet will produce a dipolar kick as seen before and therefore will affect the beam orbit.

TABLE I. Number of flux jumps in the magnetic field needed to get a $1 \%$ emittance blowup and emittance growth in 20 minutes (approximate duration of an energy ramp) at a rate of 4.4 jumps/s rate for injection optics and $\beta^{*}=1 \mathrm{~m}$.

\begin{tabular}{lccc}
\hline \hline Optics & Plane & No. of events & \multicolumn{2}{c}{$\Delta \epsilon / \epsilon$ in 20 min } \\
for $1 \% / \epsilon\left[10^{5}\right]$ & at 4.4 jumps/s [\%o] \\
\hline Injection & Horizontal & 14238 & 0.00004 \\
& Vertical & 206 & 0.00256 \\
\multirow{2}{*}{$\beta^{*}=1 \mathrm{~m}$} & Horizontal & 324 & 0.00163 \\
& Vertical & 4 & 0.11815 \\
\hline \hline
\end{tabular}

The main concern is the orbit variation at the primary collimators (TCPs) which could trigger a beam dump by the machine protection system, and therefore a considerable loss of precious time for physics. Given the slow dynamics of the flux jump with respect to the revolution frequency, a fair approximation is to assume that a flux jump induces a closed orbit variation as if it would be generated by a constant kick equal to the magnitude of the flux jump. Different machine optics have a different sensitivity to kicks generated in the triplet, where the $\beta$ functions vary considerably along the cycle. The two extreme cases considered here are the injection optics and a fully squeezed round optics $\left(15 \mathrm{~cm} \beta^{*}\right)$, together with the intermediate value of $1 \mathrm{~m} \beta^{*}$ corresponding to the end of the ramp and squeeze scheme. For all optics it is

TABLE II. Number of reactions of the regulation loop to the flux jumps needed to get a $1 \%$ emittance blowup and emittance growth in 20 minutes (approximate duration of an energy ramp) at a 4.4 events/s rate for injection optics and $\beta^{*}=1 \mathrm{~m}$.

\begin{tabular}{lccc}
\hline \hline & & No. of events & $\Delta \epsilon / \epsilon$ in 20 min \\
Optics & Plane & for $1 \% \Delta \epsilon / \epsilon\left[10^{5}\right]$ & at 4.4 jumps $/ \mathrm{s} \mathrm{[ \% o]}$ \\
\hline Injection & Horizontal & 26685 & 0.00002 \\
& Vertical & 337 & 0.00157 \\
$\beta^{*}=1 \mathrm{~m}$ & Horizontal & 574 & 0.00092 \\
& Vertical & 7 & 0.07572 \\
\hline \hline
\end{tabular}


assumed a $295 \mu \mathrm{rad}$ half crossing angle horizontal in IP1 and vertical in IP5. Table III lists the biggest (with respect to plane and IP) orbit variation at the TCPs under a field error (in units of $10^{-4}$ ) of each half-quadrupole in the triplet and for a whole triplet (Q1-Q3). The values are given in beam sigma for the nominal normalised emittance of $2.5 \mu \mathrm{m}$ at $450 \mathrm{GeV}$ (for the injection optics) and $7 \mathrm{TeV}$ (for the $1 \mathrm{~m}$ and $15 \mathrm{~cm} \beta^{*}$ optics). This, multiplied by expected amplitude of the flux jumps of 0.2 units of $10^{-4}$, gives the expected flux-jump-induced orbit variation at the TCPs, also reported in Table III. Flux jumps are expected to appear more likely at low energy, i.e., for injectionlike optics, and they should stop once reached the top energy, i.e., once the optics has normally reached $1 \mathrm{~m} \beta^{*}$. For those two optics, and similarly for any intermediate one, the impact of a single flux jump in any triplet half quadrupole is unlikely to give an orbit jump at the primary collimators able to trigger a beam dump by the machine protection system. The worst case scenario when two flux jumps of 0.2 unit amplitude occurs at the same time on the two halves of Q2 would correspond to an orbit jump at the collimators of about $4 \%$ of beam sigma. For comparison, in the unrealistic case where a 0.2 unit flux jump occurs at the fully squeezed $15 \mathrm{~cm} \beta^{*}$ optics on a Q2 half magnet, the r.m.s. orbit variation at the primary collimators would be of $5.6 \%$ of beam sigma. Such a fluctuation is considered to produce noticeable beam losses at collimators, but still unlikely to trigger a beam dump. Therefore, the orbit variation induced by a flux jumps is expected to be harmless.

As for the tune noise, the average absolute effect per quadrupole can be calculated as:

TABLE III. R.m.s. closed orbit variation at HL-LHC TCPs under the effect of the expected flux jumps for each half quadrupole composing the triplet (Q1; Q2; Q3) and for a whole triplet (Q1-Q3) computed in units of beam sigma. The optics sensitivity in units of beam sigma per unit of magnetic field change is also reported.

\begin{tabular}{lccc}
\hline \hline Optics & Magnet & $\begin{array}{c}\text { Optics sensitivity } \\
{\left[\sigma / 10^{-4}\right]}\end{array}$ & $\begin{array}{c}\text { Jump-induced } \\
\text { rms orbit }\left[10^{-3} \sigma\right]\end{array}$ \\
\hline Injection & Q1 & $<0.01$ & $<2$ \\
& Q2 & 0.01 & 2 \\
& Q3 & $<0.01$ & $<2$ \\
& Q1-Q3 & 0.01 & 2 \\
$\beta^{*}=15 \mathrm{~cm}$ & Q1 & 0.06 & 12 \\
& Q2 & 0.28 & 56 \\
& Q3 & 0.18 & 36 \\
$\beta^{*}=1 \mathrm{~m}$ & Q1-Q3 & 0.48 & 96 \\
& Q1 & 0.02 & 4 \\
& Q2 & 0.11 & 22 \\
& Q3 & 0.06 & 12 \\
\hline \hline
\end{tabular}

TABLE IV. Average absolute tune error produced by flux jumps in each quadrupole of IR1 and IR5.

\begin{tabular}{rcc}
\hline \hline & Injection & $\beta^{*}=1 \mathrm{~m}$ \\
\hline \hline$\langle|\Delta Q|\rangle\left[10^{-5}\right]$ & 1.3 & 7.8 \\
\hline \hline & & \\
& $\langle\Delta Q \mid\rangle=\frac{1}{4 \pi M} \sum_{i=1}^{M}\left|\beta_{i} \Delta k_{i} L_{i}\right|$, & (11)
\end{tabular}

where $\beta_{i}$ is the $\beta$-function at the ith quadrupole, $\Delta k_{i} L_{i}$ the integrated quadrupolar field error at the ith quadrupole and $M$ the number of quadrupoles producing errors. Assuming as before a $\Delta k$ of $0.3 \times 10^{-4} k$, the effect for injection and $\beta^{*}=1 \mathrm{~m}$ optics for the tune of both planes is shown in Table IV. These number on the order of $10^{-5}$ are on the level of the tune noise produced by the power supply current stability [15] and therefore they will have no harmful effect in the HL-LHC.

\section{THE 11 T DIPOLES}

During Long Shutdown 2 of the LHC, 4 dipoles (2 per side) using the $\mathrm{Nb}_{3} \mathrm{Sn}$ technology are planned to be installed in the dispersion suppression collimators in IR7 and to be used operationally during Run 3 .

The effect of flux jumps in the dipolar field on the emittance as a one-turn kick can be calculated as:

$$
\Delta \epsilon_{1} / \epsilon=\frac{\beta_{d} \Delta \theta^{2} \gamma_{\mathrm{rel}}}{\epsilon_{\mathrm{LHC}}}
$$

where $\beta_{d}$ is the $\beta$-function (horizontal in this case) at the dipole, $\Delta \theta$ the error in bending angle, $\gamma_{\text {rel }}$ the Lorentz factor and $\epsilon_{\mathrm{LHC}}$ the design normalized emittance. The $\beta$-function across these dipoles does not change with different optics configurations, staying about $30 \mathrm{~m}$. At $3.2 \mathrm{TeV}$ energy, this one-turn emittance growth is $\Delta \epsilon_{1} / \epsilon=3 \times 10^{-4}$ one order of magnitude lower than the effect of the HL-LHC triplet, together with the fact that there are only four $11 \mathrm{~T}$ dipoles per beam against 12 triplet quadrupoles in HL-LHC. Therefore, it can be concluded that the effect of flux jumps in the $11 \mathrm{~T}$ dipoles in the emittance will also be negligible.

TABLE V. R.m.s. closed orbit variation at HL-LHC TCPs under the effect of the expected flux jumps at the $11 \mathrm{~T}$ dipoles computed in units of beam sigma. The optics sensitivity in units of beam sigma per unit of magnetic field change is also reported.

\begin{tabular}{lcc}
\hline \hline Optics & $\begin{array}{c}\text { Optics sensitivity } \\
{\left[\sigma / 10^{-4}\right]}\end{array}$ & $\begin{array}{c}\text { Jump-induced } \\
\text { rms orbit }\left[10^{-3} \sigma\right]\end{array}$ \\
\hline Injection & 0.02 & 4 \\
$\beta^{*}=15 \mathrm{~cm}$ & 0.07 & 14 \\
$\beta^{*}=1 \mathrm{~m}$ & 0.07 & 14 \\
\hline \hline
\end{tabular}


As for the orbit noise, one can do a similar analysis as for the triplet magnets. Table $\mathrm{V}$ lists the optics sensitivity and expected closed orbit variation at the primary collimators under the effect of the expected flux jumps in the $11 \mathrm{~T}$ dipoles. As for the triplet case, the probability of inducing a beam dump with such an orbit oscillation is considered to be negligible, even for the fully squeezed optics case.

\section{FLUX JUMPS IN THE FCC-HH}

The FCC-hh (Future Circular hadron-hadron Collider) [5] will use $\mathrm{Nb}_{3}$ Sn magnet technology in all the 4672 main bending dipoles. As the nominal dipole current in the FCC-hh will be $11.2 \mathrm{kA}$ [5], the 2-3 kA region (where most of the flux jump activity happens) will correspond in the FCC-hh to a beam energy of about $8.9-13.4 \mathrm{TeV}$. Therefore, similarly to the case studied for the HL-LHC, this region of high flux jump activity appears in the early energy ramp.

Studying the FCC dipoles the same way as in the previous section, it is found that at injection optics and energy $(3.3 \mathrm{TeV})$ the average one-turn emittance growth per dipole is about $4.6 \times 10^{-4}$ relative to the FCC design normalized emittance of $2.2 \mu \mathrm{m}$. This effect is greatly amplified by the large amount of dipoles producing flux jumps (4672 compared with 12 quadrupoles in the HLLHC) and the smaller revolution frequency of the FCC which will cause the flux jumps to take less turns to rise to their full amplitude (92 turns for a $30 \mathrm{~ms}$ long flux jump).

The result of Eq. (5) at injection energy and optics raises to $\Delta \epsilon_{\max } / \epsilon=1.3 \times 10^{-4}$, being 75 flux jumps per individual dipole enough to produce a $1 \%$ emittance growth. Assuming the same event rate (4.4 events/s) and ramp length of HL-LHC (20 minutes), the flux jumps during the FCC energy ramp could cause about a $70 \%$ emittance growth. To these results will have to be added the effect of any quadrupole being traversed with a nonzero orbit, as described in previous sections.

Nevertheless, to achieve the high field density needed in the FCC magnets, the use of artificial pinning centers (APC) is expected. APCs have been observed to suppress the effect of flux jumps at low fields due to reduced lowfield magnetizations, as a result of shift in pinning force curve peak to higher fields $[16,17]$.

In summary, in FCC-hh the effect of the flux jumps is likely to have an important impact on the emittance of the beam during the energy ramp. Nevertheless, FCC-hh is going to present a much stronger synchrotron radiation damping than LHC, thus, the interplay between the flux jumps emittance growth and the radiation damping, together with the effect of APCs, should be carefully studied in the future. Also, in view of these results, the impact on all beam parameters of the flux jumps in both dipoles and quadrupoles should be carefully assessed for FCC-hh.

\section{SUMMARY}

The effect of the flux jumps in the HL-LHC triplet quadrupoles and $11 \mathrm{~T}$ dipoles has been studied. Measurements of the flux jump effect in the magnetic flux of the prototype of the $11 \mathrm{~T}$ dipole have shown a relative magnetic flux error of $(0.2 \pm 0.1) \times 10^{-4}$ with a rise time of $506 \pm 165$ turns and have been extrapolated to the HLLHC triplet. Even in the pessimistic scenario, up to $4 \times 10^{5}$ flux jumps per quadrupole would be required to produce a $1 \%$ emittance growth. At a rate of 4.4 flux jumps per second, in a ramp of 20 minutes the emittance growth will be below $0.1 \%$. The reaction of the regulation loop has been found weaker than the effect on the magnetic flux itself. Therefore, the flux jumps effect on emittance and orbit should not be considered harmful in the HL-LHC. The $11 \mathrm{~T}$ dipoles that are planned to be installed in the LHC during Long Shutdown 2 will also use the $\mathrm{Nb}_{3} \mathrm{Sn}$ technology. The effect of flux jumps in these dipoles on emittance is much smaller than the effect of the HLLHC triplet quadrupoles, due to the small horizontal $\beta$ function in the region, and can therefore be neglected too. For the same reason, also the effect on closed orbit variation and consequent possibility of causing a beam dump can be neglected.

However, for the FCC-hh main dipoles this effect cannot be neglected as the large number of main dipoles with $\mathrm{Nb}_{3} \mathrm{Sn}$ technology and the lower revolution frequency are likely to increase the effect of flux jumps to produce about a $70 \%$ emittance growth during the energy ramp, ignoring the flux jumps in the quadrupoles and the effect of the radiation damping.

Therefore, flux jumps in the magnets using $\mathrm{Nb}_{3} \mathrm{Sn}$ technology are not a concern for LHC and HL-LHC, but their effect should be carefully taken into account for future high-energy colliders.

\section{ACKNOWLEDGMENTS}

Research supported by the HL-LHC project. Many thanks to Xingchen Xu, Frank Zimmermann, and Daniel Schoerling for their comments on the effect of the APCs. Also many thanks to Massimo Giovannozzi, Gianluigi Arduini, and Lucio Rossi for many corrections and general comments on this paper.

[1] O. Brüning and L. Rossi, The High Luminosity Large Hadron Collider (World Scientific, Singapore, 2015), https://www.worldscientific.com/doi/abs/10.1142/9581.

[2] High-Luminosity Large Hadron Collider (HL-LHC): Technical Design Report V. 0.1, edited by G. Apollinari, I. Bejar, O. Bruning, P. Fessia, M. Lamont, L. Rossi, and L. Tavian, CERN Yellow Reports: Monographs (CERN, Geneva, 2017); http://cds.cern.ch/record/2284929. 
[3] P. Ferracin, G. Ambrosio, M. Anerella, F. Borgnolutti, R. Bossert, D. Cheng, D. R. Dietderich, H. Felice, A. Ghosh, A. Godeke, S. Izquierdo Bermudez, P. Fessia, S. Krave, M. Juchno, J. C. Perez, L. Oberli, G. Sabbi, E. Todesco, and M. Yu, Magnet design of the $150 \mathrm{~mm}$ aperture low- $\beta$ quadrupoles for the High Luminosity LHC, IEEE Trans. Appl. Supercond. 24, 1 (2014).

[4] A. Godeke, D. Cheng, D. R. Dietderich, P. Ferracin, S. O. Prestemon, G. Sabbi, and R. M. Scanlan, Limits of NbTi and $\mathrm{Nb}_{3} \mathrm{Sn}$, and development of W\&R Bi2212 high field accelerator magnets, IEEE Trans. Appl. Supercond. 17, 1149 (2007).

[5] M. Benedikt, M. Capeans Garrido, F. Cerutti, B. Goddard, J. Gutleber, J. M. Jimenez, M. Mangano, V. Mertens, J. A. Osborne, T. Otto, J. Poole, W. Riegler, D. Schulte, L. J. Tavian, D. Tommasini, and F. Zimmermann, Future Circular Collider, CERN Technical Report No. CERN-ACC2018-0058, 2018; https://cds.cern.ch/record/2651300.

[6] W. Meissner and R. Ochsenfeld, Ein neuer effekt bei eintritt der supraleitfähigkeit, Naturwissenschaften 21, 787 (1933).

[7] D. Boussard, W. Hfle, and T. P. R. Linnecar, The LHC transverse damper (ADT) performance specification, CERN Technical Report No. SL-Note-99-055-HRF, 2000; http://cds.cern.ch/record/702559.

[8] L. Fiscarelli, Measurements and analysis of flux jumps. Presented in the 144th HiLumi WP2 meeting, Mar 2019. https://indico.cern.ch/event/803396/.

[9] M. Martino, P. Arpaia, and S. Ierardi, Impact of flux jumps on high-precision powering of $\mathrm{Nb3Sn}$ superconducting magnets, in Proc. 10th Int. Particle Accelerator Conf.
(IPAC'19) (JACoW, Geneva, 2019), pp. 1338-1340. doi: https://doi.org/10.18429/JACoW-IPAC2019-TUPMP040.

[10] M. Martino, Progress on: Impact of flux jumps of Nb3Sn magnets on power converter performance, in the 144th HiLumi WP2 meeting, Mar 2019. https://indico.cern.ch/ event/803396/.

[11] H. Grote and F. Schmidt, MAD-X: An Upgrade from MAD8 (2003), p. 4, https://cds.cern.ch/record/618496.

[12] R. Tomás, Adiabaticity of the ramping process of an ac dipole, Phys. Rev. Accel. Beams 8, 024401 (2005).

[13] P. Bryant, Beam Transfer Lines, in CAS - CERN Accelerator School : 5th General Accelerator Physics Course, Vol. 1, Jyvskyl, Finland, Sep 1992, CERN, pp. 239-258. https://cds.cern.ch/record/235242.

[14] E. Metral et al., Update of the HL-LHC operational scenarios for proton operation (2018). https://cds.cern .ch/record/2301292.

[15] D. Gamba, G. Arduini, M. Cerqueira Bastos, J. M. Coello De Portugal-Martinez Vazquez, R. De Maria, M. Giovannozzi, M. Martino, and R. Tomas Garcia, Beam dynamics requirements for HL-LHC electrical circuits, CERN Technical Report No. CERN-ACC-2017-0101, 2017; https:// cds.cern.ch/record/2298764.

[16] X. Xingchen, X. Peng, M. Rochester, J. Sumption, and J. Lee, Opportunities and challenges with APC Nb3Sn, presented at the 22nd joint Cryogenic Engineering Conference and International Cryogenic Materials Conference (CEC-ICMC 2019) (2019), https://www.cecicmc.org.

[17] X. Xu, M. D. Sumption, and X. Peng, Internally oxidized nb3sn strands with fine grain size and high critical current density, Adv. Mater. 27, 1346 (2015). 